\title{
Relatos de intoxicação humana e animal com produtos químicos de uso veterinário em Niterói, RJ
}

\author{
Reports of human and animal poisoning with veterinary chemicals in \\ Niterói, RJ
}

Jane Silva Maia Castro'

Flavio Fernando Batista Moutinho*,II,III iD
' Secretaria de Estado de Saúde, Rio de Janeiro, RJ, Brasil

" Faculdade de Veterinária, Universidade Federal Fluminense, Niterói, RJ, Brasil

II' Fundação Municipal de Saúde de Niterói, Niterói, RJ, Brasil

\footnotetext{
* E-mail: flaviomoutinho@id.uff.br
}

Recebido: 15 jan 2020 Aprovado: 22 jun 2020

\section{RESUMO}

Introdução: A segurança química visa prevenir efeitos adversos sobre humanos, animais e ambiente. Objetivo: Avaliar o uso de produtos veterinários e os relatos de intoxicação no município de Niterói, RJ. Método: Trata-se de estudo quali-quantitativo em que foram aplicados questionários e utilizada a observação participante em dois grupos populacionais, moradores de um condomínio e de uma colônia de pescadores. Resultados: Foram entrevistadas 125 pessoas, 50 no condomínio e 75 na colônia. Houve predomínio de indivíduos com nível superior no condomínio e médio na colônia. A proporção de residências com animais domésticos foi superior à brasileira nos dois grupos. 0 uso de produtos veterinários era menor na colônia e, nessa, esses não eram prescritos pelo veterinário, ao contrário do grupo condomínio. Nos relatos de intoxicações, no condomínio, os animais foram mais expostos e, na colônia, as pessoas. Houve relato de intoxicação por aldicarbe, produto de comércio proibido. Conclusões: 0 uso de produtos veterinários era corriqueiro nos grupos estudados, mesmo sem orientação veterinária. É importante a realização de ações educativas para evitar a medicação de animais sem essa prescrição. A ocorrência de intoxicação por aldicarbe (chumbinho) chama atenção por se tratar de um produto proibido e extremamente tóxico, tornando-se fundamental o poder público aumentar a fiscalização sobre o comércio irregular desse produto.

PALAVRAS-CHAVE: Substâncias Tóxicas; Animais de Companhia; Drogas Veterinárias

\begin{abstract}
Introduction: Chemical safety aims at preventing adverse effects on humans, animals and the environment. Objective: To assess this essay is aimed at assessing the use of veterinarian products and poisoning reports in the municipality of Niterói, RJ. Method: This is a qualitative-quantitative study wherein questionnaires were provided, and two population groups were surveyed: inhabitants from an apartment complex and a fishers' community. Results: 125 people were interviewed, 50 of them in the apartment complex and 75 in the community. There was a predominance of individuals with higher education in the apartment complex and high school education in the community. The number of households with pets was higher than the Brazilian average in both groups. The usage of veterinarian products was lower in the community and, there, these products were not prescribed by a vet, contrary to the practice among the group living in the apartment complex. According to the poisoning reports, animals were more exposed to poisoning at the apartment complex, while people were more at risk in the community. Conclusions: the use of veterinary products is usual in the studied groups, even without vet's guidance, as noticed in the community. It is important to carry out educational actions to avoid medication for animal without prescription. The occurrence of intoxication by aldicarb (chumbinho) draws attention because it is a prohibited and extremely toxic product, making it essential for the public authorities to increase supervision over the irregular trade of this product.
\end{abstract}

KEYWORDS: Toxic Substances; Pets; Veterinary Drugs 


\section{INTRODUÇ̃̃O}

Existe no mercado uma variedade de produtos veterinários usados no ambiente doméstico para combater animais indesejáveis como roedores, carrapatos e pulgas. Esses produtos são vendidos em lojas de produtos agropecuários e petshops e podem ser formulados sob a forma de pós, soluções, granulados e aerossóis, apresentando-se com diferentes composições químicas, toxicidade e finalidades. 0 uso inadequado desses produtos pode causar intoxicações em humanos e animais de companhia. Essas substâncias químicas são capazes de provocar a morte de espécies vivas quando utilizados, inclusive em pequenas quantidades. São "venenos" por conceito, mas o termo foi perdido no trato cotidiano das ações de controle de pragas e foi substituído pela palavra "remédio", num verdadeiro esvaziamento do significado original. São comuns a naturalização e a banalização dos riscos decorrentes dessas substâncias, refletindo interesses estratégicos de mercado ${ }^{1,2,3}$.

A segurança química é um importante conceito global, desenvolvido para assegurar a proteção da saúde, da vida e do ambiente, frente aos riscos decorrentes da produção, comercialização, uso, armazenagem, transporte, manuseio e descarte de substâncias químicas, inclusive as de uso doméstico. Assim, a segurança química requer um conjunto de estratégias para o controle e a prevenção dos efeitos adversos para o ser humano e o ambiente decorrentes dos riscos devido a agentes químicos ${ }^{4}$.

Segundo Paumgartten ${ }^{5}$, virtualmente todas as substâncias químicas podem causar efeitos adversos, dependendo da dose e das condições em que os indivíduos são a elas expostos. A toxicologia, isto é, o estudo dos efeitos danosos de substâncias químicas em organismos vivos, fornece a base de dados científicos na qual se apoia a avaliação de riscos de efeitos adversos para a saúde.

Os produtos veterinários são substâncias químicas reguladas pelo Ministério da Agricultura Pecuária e Abastecimento (MAPA), por meio do Decreto-Lei ${ }^{\circ} 467$, de 13 de fevereiro de $1969^{6}$, que estabelece os critérios para fiscalização, fracionamento, comercialização, armazenamento, manipulação, acondicionamento e utilização desses produtos, e obriga os estabelecimentos que desenvolvem essas atividades a se licenciarem no MAPA.

Já o Decreto $n^{\circ} 8.840$, de 24 de agosto de $2016^{7}$, que regulamenta a legislação sobre a fabricação e a comercialização de produtos veterinários, define produto veterinário como:

toda substância química, biológica, biotecnológica ou preparação manufaturada cuja administração seja aplicada de forma individual ou coletiva, direta ou misturada com os alimentos, destinada à prevenção, ao diagnóstico, à cura ou ao tratamento das doenças dos animais, incluindo os aditivos, suplementos promotores, melhoradores da produção animal, medicamentos, vacinas, antissépticos, desinfetantes de ambiente e de equipamentos, pesticidas e todos os produtos que, utilizados nos animais ou no seu habitat, protejam, restaurem ou modifiquem suas funções orgânicas e fisiológicas, ou também os produtos destinados ao embelezamento dos animais.

A sociedade brasileira vem passando por mudanças significativas, com redução do tamanho das famílias e novos estilos de vida, o que vem levando ao aumento da procura de cães e gatos de estimação ${ }^{8}$.

A presença de animais de companhia nos lares brasileiros é expressiva. Estima-se que em $44,3 \%$ deles haja pelo menos um cachorro, percentual que chega a $65,0 \%$ na zona rural. 0 total de cães de companhia chegaria a 52,2 milhões, ou 1,8 cachorro por domicílio. Já em relação aos gatos, a estimativa é de que $17,7 \%$ dos domicílios brasileiros teria ao menos um gato, proporção que também aumenta consideravelmente na zona rural, onde alcança $38,4 \%$ dos lares. A população estimada de felinos de companhia seria de 22,1 milhões de animais, ou 1,9 gato por domicílio?.

Em face do exposto, o presente trabalho teve como objetivo avaliar os hábitos de uso de produtos veterinários e os relatos de intoxicação de humanos e animais moradores do município de Niterói, RJ.

\section{MÉTODO}

A presente pesquisa foi desenvolvida na Região Oceânica do município de Niterói, que está localizado na região metropolitana do estado do Rio de Janeiro, com uma área territorial de $33.919 \mathrm{~km}^{2}$, localizada entre a Baía de Guanabara ao Oeste, o Oceano Atlântico ao Sul, a cidade de Maricá ao Leste e a cidade de São Gonçalo ao Norte. O município possuía população estimada para 2017 de 499.028 habitantes $^{10}$. A Região Oceânica de Niterói foi escolhida para a pesquisa devido ao seu crescimento desordenado envolvendo populações de diferentes estratos sociais e degradação ambiental ${ }^{11}$.

Trata-se de um estudo quali-quantitativo em que foram adotadas as seguintes estratégias: observação participante e aplicação de questionários com perguntas fechadas e abertas. A pesquisa de campo foi realizada em 2010 por intermédio de visitas domiciliares na área de estudo e englobou dois grupos de entrevistados:

1. Condomínio de classe média alta: para a composição da amostragem do "Grupo Condomínio", foi adotado o procedimento de amostragem sistemática em população aleatória, no qual, a cada quatro casas, foi realizada uma entrevista. Foram investigadas 50 residências das 232 existentes no condomínio.

2. Moradores cadastrados pelo Programa Médico de Família da Colônia de Pescadores de Itaipu: esse cadastramento implica condição econômica de baixa renda. O módulo "Colônia de Pescadores" possuía 371 residências cadastradas e participaram da amostra 75 destas. 
O critério de inclusão para participação na pesquisa foi ter ao menos 18 anos e de exclusão ser menor de 18 anos. A aplicação do questionário por domicílio teve duração média de 15 a $20 \mathrm{~min}$.

Os dados foram tabulados com técnicas de estatística descritiva e foram calculadas as frequências relativa e absoluta dos dados quantitativos por meio do programa Microsoft ${ }^{\oplus}$ Office Excel 2003.

A pesquisa foi aprovada pelo Comitê de Ética da Escola Nacional de Saúde Pública da Fundação Oswaldo Cruz (ENSP/Fiocruz) (CAA 00210031000-09), atendendo às normas do Conselho Nacional de Ética e Pesquisa. Aos entrevistados foi solicitada uma autorização formal, através de assinatura do Termo de Consentimento Livre e Esclarecido, após os esclarecimentos sobre os objetivos da pesquisa.

\section{RESULTADOS E DISCUSSÃO}

\section{Perfil social e econômico dos entrevistados}

Foram entrevistadas 125 pessoas, sendo 50 no condomínio de classe média alta (renda média acima de 10 salários mínimos) e 75 em residências cadastradas no módulo médico de família da Colônia de Pescadores de Itaipu (renda média de 1 a 3 salários mínimos).

Do primeiro grupo, denominado ao longo da pesquisa como Grupo Condomínio, $72 \%$ dos entrevistados eram do sexo feminino e $28 \%$ do sexo masculino. No segundo grupo, denominado Grupo Colônia de Pescadores, $77,3 \%$ eram do sexo feminino e $22,7 \%$ do sexo masculino.

Quanto ao estado civil, 40,0\% eram casados e $26,0 \%$ solteiros, no Grupo Condomínio; e $38,7 \%$ eram casados e $40,0 \%$ solteiros no Grupo Colônia de Pescadores.

Em relação à faixa etária, observou-se que, no Grupo Condomínio, $22,0 \%$ estavam entre 50 a 59 anos, e $18,0 \%$ na faixa de 40 a 49 anos. No Grupo Colônia de Pescadores, 33,0\% se situavam na faixa de 50 a 59 anos, e $26,7 \%$ na faixa de 40 a 49 anos.

Em relação à escolaridade, 52,0\% do Grupo Condomínio tinham ensino superior completo, e $20,0 \%$ possuíam ensino médio completo. No Grupo Colônia de Pescadores, 40,0\% possuíam ensino médio completo e $3,06 \%$ ensino fundamental incompleto, o que caracterizou as diferenças sociais de renda e escolaridade entre os dois grupos.

Em relação à presença de crianças, observou-se a sua presença em 36,0\% das casas do Grupo Colônia de Pescador, enquanto no Grupo Condomínio, elas foram encontradas em $24,0 \%$ das residências.

Domicílios com animais de companhia

No tocante a animais de estimação, só 8,0\% das casas do Grupo Condomínio não os possuíam, enquanto no Grupo Colônia de Pescadores, 36,0\% não residiam com nenhum animal de estimação. Esses dados demonstram uma maior proporção de residências com presença de animais de estimação do que a estimativa para o Brasil para ambos os grupos, quando comparados com as estimativas do Instituto Brasileiro de Geografia e Estatística (IBGE) 9 . 0 cão era a espécie mais presente nos dois grupos: $80,0 \%$ no Grupo Condomínio e 60,0\% no Grupo Colônia de Pescadores, onde, em muitos casos, eram criados soltos. A presença maior do cão nos lares encontra respaldo na pesquisa desenvolvida pelo IBGE 9 em nível nacional, quando foi encontrado grande predomínio da presença de cães.

\section{Uso de produtos veterinários}

Quanto ao uso de produtos veterinários, o Grupo Colônia de Pescadores apresentou uma menor percentagem quando comparado ao Grupo Condomínio, possivelmente por questões econômicas. Outra diferença foi em relação à prescrição dos produtos: para o Grupo Colônia de Pescadores, a aquisição dos produtos ocorria por indicação de pessoa sem conhecimento técnico (balconista da loja); o Grupo Condomínio recebia a prescrição de profissional médico veterinário. Neste aspecto, o grupo pesquisado de maior renda utilizava maior quantidade e variedade de produtos veterinários do que o grupo de menor renda. Esta inferência está de acordo com o estudo realizado por Davis et al. ${ }^{12}$, no estado do Missouri, EUA. A motivação para o uso desses produtos ocorria em função do controle de pulgas e carrapatos em animais domésticos como cães e gatos.

Nas Tabelas 1 e 2 foram descritos os produtos veterinários encontrados respectivamente nas residências do Grupo Condomínio e do Grupo Colônia de Pescadores, sua ocorrência, classe toxicológica e seu principal grupo químico. Cabe destacar que foram elencados somente os produtos usados para controlar ectoparasitas e endoparasitas dos animais de companhia.

\section{Relatos de casos de intoxicação}

Quando foi perguntado aos entrevistados sobre a existência pregressa de casos de intoxicação por produto químico de uso veterinário, 70,0\% $(n=35)$ dos entrevistados do Grupo Condomínio relataram que não havia, $10,0 \%(n=5)$ não informaram, $18,0 \%(n=9)$ relataram intoxicação em animais e $2,0 \%(n=1)$ em humanos. Os produtos veterinários causadores das intoxicações em animais foram: Butox ${ }^{\circledast}$ (dois casos), Frontline ${ }^{\circledast}$ (dois casos) e Creolina (um caso). Foram relatadas, ainda, quatro intoxicações por "chumbinho" (aldicarbe). A intoxicação em humano aconteceu por Butox ${ }^{\circledR}$ e o entrevistado relatou não ter se protegido adequadamente no momento da aplicação do produto no ambiente.

Já no Grupo Colônia de Pescadores, 93,4\% $(n=70)$ dos entrevistados afirmaram não terem sofrido intoxicação por produto químico de uso veterinário, 4,0\% $(\mathrm{n}=3)$ não informaram, $1,3 \%(n=1)$ relataram intoxicação em animal (por coleira impregnada de inseticida) e $1,3 \%(n=1)$ relataram intoxicação humana por Triatox ${ }^{\circledR}$. Esses dados condizem com a menor quantidade de produtos químicos de uso veterinário encontrados nas residências do Grupo Colônia de Pescadores em relação ao Grupo Condomínio. 
Tabela 1. Produtos veterinários encontrados nas residências do Grupo Condomínio, Niterói, RJ, 2010.

\begin{tabular}{|c|c|c|c|c|c|}
\hline Nome comercial & $\begin{array}{l}\text { Ocorrência } \\
\left(\mathrm{N}^{\circ}\right)\end{array}$ & Percentual (\%) & $\begin{array}{l}\text { Principal grupo } \\
\text { químico }\end{array}$ & Tipo classe produto & Classe toxicológica \\
\hline Frontline $^{\circledast}$ & 24 & 48,0 & Fipronil & Ectoparasiticida & IV \\
\hline Butox $^{\circledast}$ & 16 & 32,0 & Piretroide & Ectoparasiticida & IV \\
\hline $\begin{array}{l}\text { Sabonete/ Shampoo uso } \\
\text { veterinário }\end{array}$ & 13 & 26,0 & * & * & * \\
\hline Kiltrix ${ }^{\oplus}$ & 11 & 22,0 & Carbamato & Ectoparasiticida & III \\
\hline Sabonete Assuntol & 6 & 12,0 & $\begin{array}{l}\text { Organofosforado } \\
\text { (coumafós) }\end{array}$ & Ectoparasiticida & II \\
\hline K-othrine ${ }^{\circledast}$ & 2 & 4,0 & Piretróide & Inseticida & IV \\
\hline Triatox ${ }^{\circledast}$ & 2 & 4,0 & Amitraz & $\begin{array}{l}\text { Inseticida } \\
\text { Acaricida }\end{array}$ & III \\
\hline Advantage $^{\oplus}$ & 1 & 2,0 & Imidacloprida & Endoparasiticida & $* *$ \\
\hline Capstar $^{\oplus}$ & 1 & 2,0 & Nitenpyran & Endoparasiticida & $* *$ \\
\hline Fiprolex ${ }^{\circledast}$ & 1 & 2,0 & Fipronil & Ectoparasiticida & II \\
\hline Program $^{\oplus}$ & 1 & 2,0 & $\begin{array}{l}\text { Lufenuron } \\
\text { Milbemicina }\end{array}$ & Endoparasiticida & III \\
\hline Promeris $^{\circledast}$ & 1 & 2,0 & Metaflumizona & Ectoparasiticida & IV \\
\hline Revolution $^{\oplus}$ & 1 & 2,0 & Selamectina & Endoparasiticida & III \\
\hline Sabonete Tetsarnol ${ }^{\circledast}$ & 1 & 2,0 & Monossulfiram & Ectoparasiticida & $* *$ \\
\hline Shampoo Ecovet ${ }^{\oplus}$ & 1 & 2,0 & Piretroide & Ectoparasiticida & III \\
\hline Topline ${ }^{\circledast}$ & 1 & 2,0 & Fipronil & Ectoparasiticida & II \\
\hline
\end{tabular}

Fonte: Elaborada pelos autores, 2020.

* Não informou o nome do produto.

** Medicamento veterinário.

Tabela 2. Produtos veterinários utilizados pelo Grupo Colônia de Pescadores, Niterói, RJ, 2010.

\begin{tabular}{|c|c|c|c|c|c|}
\hline Nome comercial & $\begin{array}{c}\text { Ocorrência } \\
\left(\mathrm{N}^{\circ}\right)\end{array}$ & Percentual (\%) & $\begin{array}{l}\text { Principal grupo } \\
\text { químico }\end{array}$ & Tipo classe produto & Classe toxicológica \\
\hline Sabonete/ Shampoo & 12 & 16,0 & * & * & * \\
\hline K-othrine ${ }^{\circledast}$ & 3 & 4,0 & Piretroide & Inseticida & IV \\
\hline Comprimido antiparasita & 2 & 2,7 & * & * & * \\
\hline Triatox ${ }^{\circledast}$ & 2 & 2,7 & Amitraz & Acaricida & III \\
\hline Talco antiparasita & 1 & 1,3 & * & * & * \\
\hline Spray cicatrizante & 1 & 1,3 & * & * & * \\
\hline Sabonete Assuntol & 1 & 1,3 & Organofosforado & Ectoparasiticida & II \\
\hline Capstar $^{\oplus}$ & 1 & 1,3 & Nitenpyran & Endoparasiticid & $* *$ \\
\hline Creolina $^{\oplus}$ no animal & 1 & 1,3 & Cresol & Desinfetante & \# \\
\hline Neocid $^{\circledast}$ no animal & 1 & 1,3 & Carbamato & Inseticida & II \\
\hline
\end{tabular}

Fonte: Elaborada pelos autores, 2020.

* Não informou o nome do produto.

** Medicamento Veterinário.

\# Não encontrado.

A regulação dos produtos de uso veterinário é realizada pelo MAPA e muitos produtos possuem componentes químicos na sua formulação que são tóxicos para o ambiente e para várias espécies, inclusive a nossa. A maioria das bulas e rótulos dos produtos de uso veterinário descreve os efeitos colaterais, sua composição química, mas não informa a classe toxicológica e nem o seu grau de risco para o ambiente. Nesta pesquisa foram evidenciados os relatos de casos de intoxicações por produtos de uso veterinário relatados pelos entrevistados.

Os produtos referidos que causaram intoxicação em humanos foram o Triatox ${ }^{\oplus}$ e o Butox ${ }^{\circledast}$, corroborando o estudo feito por Silva et al. ${ }^{13}$, no município de Cáceres, Mato Grosso, o qual constatou que há venenos que são indicados no combate de pragas 
agrícolas e que, no entanto, são usados no ambiente doméstico como controladores de pragas, com elevado risco de dano à vida dos aplicadores. No trabalho em questão, foi relatado o uso do aldicarbe que, apesar de ser um produto de uso agrícola, é comumente utilizado ilegalmente no controle de roedores no Brasil e, por isso, optou-se por incluir no presente trabalho esse resultado, já que causa intoxicações em animais e pode causar em humanos.

Segundo o Sistema Nacional de Informações Tóxico-Farmacológicas (Sinitox), em 2017 foram 711 casos de intoxicação humana e 109 casos de intoxicação animal por produtos veterinários ${ }^{14}$. Diversos trabalhos vêm mostrando um percentual muito baixo de intoxicação por produtos veterinários no Brasil ${ }^{15,16}$. Mas a notificação dos casos de intoxicação por produtos veterinários é necessária e habitualmente realizada de modo precário, gerando subnotificação. As causas da subnotificação podem ser muitas, entre elas está o fato de a contaminação por substâncias químicas muitas vezes não ser percebida pela população. Por não serem graves, muitas vezes as intoxicações não exigem internação do indivíduo, ou sequer geram a procura de atendimento médico. A população, de uma forma geral, desconhece a importância desta atitude, que permitiria o nexo associativo entre os sintomas e os agentes químicos ${ }^{17}$.

Estudo desenvolvido por Lira et al. ${ }^{18}$, em Fortaleza, no Ceará, avaliando a intoxicação de crianças e adolescentes, mostrou que o principal grupo de agentes tóxicos envolvidos foi o dos raticidas (incluindo aí o "chumbinho" - aldicarbe - utilizado ilegalmente no controle de roedores). Mas esse estudo mostrou, também, considerável participação dos produtos veterinários nas intoxicações, variando de $2,5 \%$ dos casos na faixa etária de 12 a 16 anos, a $9,1 \%$ na de 0 a 4 anos.

Os carbamatos vêm aparecendo em diversos estudos sobre intoxicações de animais domésticos como a principal substância envolvida. Estudo de Medeiros et al. ${ }^{19}$, ao analisarem os casos de intoxicação exógena de cães e gatos atendidos na Faculdade de Veterinária da Universidade Federal Fluminense, em Niterói, no período 2002 a 2008, encontrou predomínio das intoxicações medicamentosas, seguidas do uso de "chumbinho" (carbamato). Estudo desenvolvido por Menezes et al. ${ }^{20}$ encontrou em cães necropsiados mortos por intoxicação predomínio $(47,0 \%$ ou $8 / 17)$ dos carbamatos nos exames toxicológicos desenvolvidos. Estudo desenvolvido por Bulcão et al. ${ }^{21}$, no Rio Grande do Sul, mostrou ser o aldicarbe o principal agente tóxico envolvido, respondendo por $39,7 \%(n=27)$ das intoxicações de animais domésticos.

Esses dados contundentes mostram falha na fiscalização da comercialização do aldicarbe, já que se tratava de produto de uso agrícola cuja venda demanda receituário agronômico. Além disso, o uso de raticidas agudos é proibido no país, em função do alto risco à saúde ${ }^{22}$. Desde 2012, é proibida a produção, o comércio e o uso de produtos à base de aldicarbe no Brasil, sendo que a licença do último produto que ainda era produzido em território nacional foi cancelada pelo MAPA naquele ano ${ }^{23}$. Apesar disso, o relatório do Programa de Análise de Resíduos de Agrotóxicos em
Alimentos (2013-2015) ainda mostrava a presença de aldicarbe em goiabas e uvas ${ }^{24}$, o que indica a possível entrada clandestina do produto no Brasil.

Souza et al. ${ }^{25}$ chamaram atenção para o risco da intoxicação de animais domésticos pela desinformação dos tutores ao tentarem medicar seus animais ou eliminar roedores ou insetos sem consultar um profissional da área. Como foi visto, isso ocorreu no grupo Colônia de Pescadores. Estudo desenvolvido por Zielke et al. ${ }^{26}$ reforça essa preocupação ao concluir que o uso indiscriminado e sem prescrição de ectoparasiticidas veterinários é frequente entre os tutores de cães e gatos.

Atualmente, vem sendo observado aumento na quantidade de acidentes envolvendo os produtos químicos de uso veterinário, especialmente na área urbana e, principalmente, envolvendo a população de menor escolaridade, o que pode estar relacionado à dificuldade na compreensão das instruções de manejo dos produtos ${ }^{27}$. Importante destacar que, a despeito disso, pesquisa desenvolvida por Barboza et al. ${ }^{28}$ no Rio de Janeiro, mostrou que $33,4 \%$ dos entrevistados identificavam os produtos veterinários como substâncias potencialmente intoxicantes.

Vale ressaltar que os produtos veterinários para o controle de ectoparasitas, raticidas, pulgicidas, mesmo de classe toxicológica de risco alto como os carbamatos e os organofosforados são vendidos nos petshops e nas lojas de produtos agropecuários sem receituário médico veterinário.

Por fim, cabe destacar a dificuldade que os próprios médicos veterinários têm em conhecer a relação de produtos veterinários licenciados pelo MAPA. Em 2016 o Conselho Federal de Medicina Veterinária oficiou pela segunda vez aquele ministério uma solicitação sobre a divulgação da lista de medicamentos veterinários com registro válido junto ao órgão, dada a dificuldade de os médicos veterinários terem acesso a essa informação ${ }^{29}$.

\section{CONCLUSÕES}

Pode-se concluir que o uso de produtos químicos veterinários é corriqueiro nos grupos estudados e que as pessoas com menor renda os adquirem sem orientação do médico veterinário, logo, eles são vendidos sem prescrição ou receituário.

É importante a realização de ações educativas junto aos tutores de animais visando sensibilizá-los para a importância de não medicar os animais sem consultar um médico veterinário bem como sobre atentar para as informações presentes nas bulas dos produtos de uso veterinário, principalmente daqueles destinados ao controle de ectoparasitas, que foram os principais causadores de intoxicação.

A ocorrência de intoxicação por aldicarbe chama atenção por se tratar de um produto proibido e extremamente tóxico.

Torna-se fundamental que o poder público aumente a fiscalização sobre o comércio irregular desse produto a fim de salvaguardar a saúde coletiva. 
1. Augusto LGS, Novaes TCP, Furtado A, Abrahão CEC, Souza CA, Pavão AC. Avaliação crítica do programa de erradicação do Aedes aegypti: contribuições técnicas para medidas de controle. Rev IMIP. 2000;14(1):90-7.

2. Castro JSM. Práticas de uso de agrotóxicos no município de Cachoeiras de Macacu, RJ: um estudo de saúde ambiental [dissertação]. Niterói: Universidade Federal Fluminense; 1999.

3. Peres F, Rozember B, Alves SR, Moreira JS, Oliveira-Silva JJ. Comunicação relacionada ao uso de agrotóxicos em região agrícola do estado do Rio de Janeiro. Rev Saude Publica. 2001;35(6):564-70. https://doi.org/10.1590/S0034-89102001000600010

4. Pivetta F, Porto MFS, Machado JHM, Moreira JC, Freitas CM, Furtado JS. Perspectivas para a formulação de uma política nacional de segurança química no Brasil. In: $2^{\circ}$ Seminário Nacional de Saúde e Ambiente e Desenvolvimento; Rio de Janeiro, Brasil. Rio de Janeiro: Fundação Oswaldo Cruz; 2002.

5. Paumgartten FJR. Risk assessment for chemical substances: the link between toxicology and public health. Cad Saude Publica. 1993;9(4):439-47. https://doi.org/10.1590/S0102-311X1993000400004

6. Brasil. Decreto Lei $\mathrm{N}^{\circ} 467$, de 13 de fevereiro de 1969. Dispõe sobre a fiscalização de produtos de uso veterinário, dos estabelecimentos que os fabriquem e dá outras providências. Diário Oficial União.14 fev 1969.

7. Brasil. Decreto $N^{\circ} 8.840$, de 24 de agosto de 2016 . Altera o anexo ao decreto $\mathrm{N}^{\circ} 5.053$, de 22 de abril de 2004, que aprova o regulamento de fiscalização de produtos de uso veterinário e dos estabelecimentos que os fabriquem ou comerciem. Diário Oficial União. 25 ago 2016.

8. Silva MHS, Silva JA, Magalhães DF, Silva MX, Meneses JMC, Moreira EC. Caracterização demográfica e epidemiológica de cães e gatos domiciliados em Barbacena, MG. Arq Bras Med Vet Zootec. 2010;62(4):1002-6. https: / /doi.org/10.1590/S0102-09352010000400035

9. Instituto Brasileiro de Geografia e Estatística - IBGE. Pesquisa nacional de saúde 2013: acesso e utilização dos serviços de saúde, acidentes e violências. Rio de Janeiro: Instituto Brasileiro de Geografia e Estatística; 2015.

10. Brasileiro de Geografia e Estatística - IBGE. Rio de Janeiro: cidades: Niterói. Rio de Janeiro: Instituto Brasileiro de Geografia e Estatística; 2017[acesso 16 maio 2018]. Disponível em: https://cidades.ibge.gov.br/brasil/rj/ niteroi/panorama

11. Rocha AM, Guerra AJT, Santos AG, Oliveira AC, Neves FO. Diagnóstico da degradação ambiental no bairro de Itaipu, Niterói, RJ. In: $7^{\circ}$ Simpósio Nacional de Controle de Erosão; Goiânia, Brasil. São Paulo: Associação Brasileira de Geologia de Engenharia e Ambiental; 2001.
12. Davis JR, Brownson RC, Garcia R. Family pesticide use in the home, garden, orchard, and yard. Arch Environ Contam Toxicol.1992;22:260-6. https://doi.org/10.1007/BF00212083

13. Silva A, Rieder A, Dores EFGC, Rodrigues GL, Mendes MF, Silva PL et al. Agentes pesticidas causadores de intoxicação em três zonas habitacionais do município de Cáceres, Alto pantanal, MT, Brasil. In: $4^{\circ}$ Simpósio sobre Recursos Naturais e Sócio-Econômico do Pantanal; Corumbá, Brasil. Brasília: Empresa Brasileira de Pesquisa Agropecuária; 2004.

14. Fundação Oswaldo Cruz - Fiocruz. Sistema nacional de informações tóxico-farmacológicas (Sinitox). Rio de Janeiro: Fundação Oswaldo Cruz; 2017[acesso 6 maio 2020]. Disponível em: http://www.fiocruz.br/sinitox_novo/cgi/ cgilua.exe/sys/start.htm?sid=4

15. Burity RAB, Ribeiro JSD, Guimarães ES, Freitas JM, Freitas MTD, Lima GVPS et al. Perfil epidemiológico das intoxicações exógenas no município de Moreno, PE no período de 2012 a 2015. Med Vet. 2019;13(1):49-56. https://doi.org/10.26605/medvet-v13n1-2609

16. Amorim MLP, Mello MJG, Siqueira MT. Intoxicações em crianças e adolescentes notificados em um centro de toxicologia no nordeste do Brasil. Rev Bras Saude Matern Infant. 2017;17(4):773-80. https://doi.org/10.1590/1806-93042017000400009

17. Augusto LGS. Saúde e vigilância ambiental: um tema em construção. Epidemiol Serv Saude. 2003;12(4):177-86. https://doi.org/10.5123/S1679-49742003000400002

18. Lira SVM, Silva JG, Abreu RNDC, Moreira DP, Vieira LJES, Frota MA. Intoxicações por pesticidas em crianças, adolescentes e jovens no município de Fortaleza (CE). Cienc Cuid Saude. 2009;8(1):48-55. https://doi.org/10.4025/cienccuidsaude.v8i1.7772

19. Medeiros RJ, Monteiro FO, Silva GC, Nascimento Júnior A. Casos de intoxicações exógenas em cães e gatos atendidos na Faculdade de Veterinária da Universidade Federal Fluminense durante o período de 2002 a 2008. Cienc Rural. 2009;39(7):2105-10. https://doi.org/10.1590/S0103-84782009005000151

20. Menezes RS, Almeida ECP, Ferreira AMR. Análise retrospectiva de casos de intoxicação fatal em cães e gatos diagnosticados em necropsias no período de 2004 a 2011. In: Congresso Medvep de Especialidades Veterinárias; Curitiba, Brasil. Curitiba: Medvep; 2011.

21. Bulcão RP, Tonello R, Piva SJ, Schmitt GC, Emanuelli T, Dallegrave E et al. Intoxicação em cães e gatos: diagnóstico toxicológico empregando cromatografia em camada delgada e cromatografia líquida de alta pressão com detecção ultravioleta em amostras estomacais. Cienc Rural. 2010;40(5):1109-13. https://doi.org/10.1590/S0103-84782010000500017

22. Fundação Nacional de Saúde - Funasa. Manual de controle de roedores. Brasília: Fundação Nacional de Saúde; 2002. 
23. Agência Nacional de Vigilância Sanitária - Anvisa. Anvisa esclarece. Brasília: Agência Nacional de Vigilância Sanitária; 2016[acesso 16 maio 2018]. Disponível em: http://portal.anvisa.gov.br/anvisaesclarece?p_p_id=baseconhecimentoportlet_WAR_ baseconhecimentoportlet\&p_p_lifecycle=0\&p_p_ state=normal\&p_p_mode=view\&p_p_col_id=column-2\&p_p_ col_pos=1\&p_P_col_count=2\&_baseconhecimentoportlet_ WAR_baseconhecimentoportlet_assuntold $=7 \&$ _ baseconhecimentoportlet_WAR_baseconhecimentoportlet_ conteudold=0\&_baseconhecimentoportlet_WAR_ baseconhecimentoportlet_view=detalhamentos

24. Agência Nacional de Vigilância Sanitária - Anvisa. Programa de análise de resíduos de agrotóxicos em alimentos: relatório das análises de amostras monitoradas no período de 2013 a 2015. Brasília: Agência Nacional de Vigilância Sanitária; 2016.

25. Souza DG, Oliveira MS, Torres APC. Intoxicação medicamentosa em animais. Rev Conexão Eletrônica. 2017;14(1):327-32.
26. Zielke M, Carvalho LF, Salame JP, Barboza DV, Gaspar LFJ, Sampaio LCL. Avaliação do uso de fármacos em animais de companhia sem orientação profissional. Sci Ani Heal. 2018;6(1):29-46. https://doi.org/10.15210/sah.v6i1.13184

27. Duarte PM, Santana VTP, Dalmas AD. Perfil epidemiológico das intoxicações por produtos veterinários no estado do Rio Grande do Sul entre 2007 e 2017. Connect Line. 2019;(21):147-63. https://doi.org/10.18312/2Fconnectionline.v0i21.1404

28. Barboza FS, Gomes BR, Moreira IO, Pereira LC, Pinto YF, Silva JCS. Conhecimento dos riscos e circunstâncias dos envenenamentos. Rev Saude Fis Ment. 2017;5(2):6-17.

29. Conselho Federal de Medicina Veterinária CFMV. CFMV solicita ao MAPA divulgação da lista atualizada de medicamentos de uso veterinário com registro válido. Notícias. 26 out 2016[acesso 16 maio 2018]. Disponível em: http://portal.cfmv.gov.br/noticia/index/id/4882

Contribuição dos autores

Maia J - Concepção, planejamento (desenho do estudo), aquisição, análise, interpretação dos dados e redação do trabalho. Moutinho FFB - Análise, interpretação dos dados e redação do trabalho. Todos os autores aprovaram a versão final do trabalho.

Conflito de Interesse

Os autores informam não haver qualquer potencial conflito de interesse com pares e instituições, políticos ou financeiros deste estudo.

Licença CC BY-NC atribuição não comercial. Com essa licença é permitido acessar, baixar (download), copiar, imprimir, compartilhar, reutilizar e distribuir os artigos, desde que para uso não comercial e com a citação da fonte, conferindo os devidos créditos de autoria e menção à Visa em Debate. Nesses casos, nenhuma permissão é necessária por parte dos autores ou dos editores. 